\title{
Somatostatin Receptor Imaging and Theranostics: Current Practice and Future Prospects
}

Sonya Park ${ }^{1}$, Ashwin Singh Parihar ${ }^{2,3}$, Lisa Bodei $^{4}$, Thomas A. Hope ${ }^{5}$, Nadine Mallak $^{6}$, Corina Millo ${ }^{7}$, Kalpna Prasad $^{8}$, Don Wilson ${ }^{9,10}$, Katherine Zukotynski ${ }^{11-13}$, and Erik Mittra ${ }^{6}$

${ }^{I}$ Department of Nuclear Medicine, Seoul St. Mary's Hospital, Seoul, Korea $;{ }^{2}$ Department of Nuclear Medicine, Postgraduate Institute of Medical Education and Research, Chandigarh, India; ${ }^{3}$ Mallinckrodt Institute of Radiology, Washington University School of Medicine, St. Louis, Missouri; ${ }^{4}$ Molecular Imaging and Therapy Service, Memorial Sloan Kettering Cancer Center, New York, New York; ${ }^{5}$ Department of Radiology and Biomedical Imaging, University of California San Francisco, San Francisco, California; ${ }^{6}$ Department of Diagnostic Radiology, Oregon Health \& Science University, Portland, Oregon; ${ }^{7}$ Department of Nuclear Medicine, RAD\&IS, Clinical Center, National Institutes of Health, Bethesda, Maryland; ${ }^{8}$ Department of Nuclear Medicine, Walter Reed National Military Medical Center, Bethesda, Maryland; ${ }^{9}$ BC Cancer, Vancouver, British Columbia, Canada; ${ }^{10}$ Department of Radiology, University of British Columbia, Vancouver, British Columbia, Canada; ${ }^{11}$ Departments of Radiology and Medicine, McMaster University, Hamilton, Ontario, Canada; ${ }^{12}$ Department of Medical Imaging, Schulich School of Medicine \& Dentistry, Western University, London, Ontario, Canada; and ${ }^{13}$ Department of Radiology, University of British Columbia, Vancouver, British Columbia, Canada

A new era of precision diagnostics and therapy for patients with neuroendocrine neoplasms began with the approval of somatostatin receptor (SSTR) radiopharmaceuticals for PET imaging followed by peptide receptor radionuclide therapy (PRRT). With the transition from SSTRbased $\gamma$-scintigraphy to PET, the higher sensitivity of the latter raised questions regarding the direct application of the planar scintigraphy-based Krenning score for PRRT eligibility. Also, to date, the role of SSTR PET in response assessment and predicting outcome remains under evaluation. In this comprehensive review article, we discuss the current role of SSTR PET in all aspects of neuroendocrine neoplasms, including its relation to conventional imaging, selection of patients for PRRT, and the current understanding of SSTR PET-based response assessment. We also provide a standardized reporting template for SSTR PET with a brief discussion.

Key Words: somatostatin; SSTR; peptide receptor radionuclide therapy; neuroendocrine neoplasms; ${ }^{68} \mathrm{Ga}$-DOTATATE; ${ }^{68} \mathrm{Ga}$-DOTANOC

J Nucl Med 2021; 62:1323-1329

DOI: 10.2967/jnumed.120.251512

$\mathbf{N}$ euroendocrine neoplasms (NENs) are rare, heterogeneous, and typically slow-growing, accounting for about $0.5 \%$ of all diagnosed malignancies. Originating from the secretory cells of the neuroendocrine system at almost any anatomic site, their site of origin is often linked to disease biology. For example, tumors of the ileum typically have a high malignant potential, although metastatic lesions tend to have an indolent course. Gastric and rectal tumors have a low metastatic potential but can grow aggressively once metastatic (1). Gastroenteropancreatic, pulmonary, and thymic NENs are among the most commonly diagnosed (2). The term NENs encompasses both well-differentiated neuroendocrine tumors (NETs) and poorly differentiated neuroendocrine carcinomas.

Received Feb. 5, 2021; revision accepted Jun. 29, 2021.

For correspondence or reprints, contact Erik Mittra (mittra@ohsu.edu). Published online July 22, 2021.

COPYRIGHT (C) 2021 by the Society of Nuclear Medicine and Molecular Imaging.
Whereas neuroendocrine carcinomas are of high grade by default, NETs are classified further according to histologic grade and degree of differentiation, with site-specific parameters (cutoffs). Grading for gastroenteropancreatic NETs, for example, is based on proliferation using either the Ki-67 index or mitotic count per 10 high-power fields. Grade 1 (G1 or low-grade) refers to a Ki-67 of less than 3\% and fewer than 2 mitoses per 10 high-power fields, G2 refers to a Ki-67 of 3\%-20\% or 2-20 mitoses per 10 high-power fields, and G3 refers to a Ki-67 of more than $20 \%$ or more than 20 mitoses per 10 high-power fields (3). On the basis of the degree of differentiation, they are categorized as either well-differentiated or poorly differentiated tumors. Most NENs are sporadic, although some arise in the setting of inherited syndromes such as multiple endocrine neoplasia, tuberous sclerosis, Von Hippel-Lindau disease, or neurofibromatosis (1).

NENs typically have increased expression of somatostatin receptors (SSTRs), which are G-protein-coupled receptors modulating cellular proliferative and secretory activity. This expression forms the basis of functional imaging with SSTR-targeting radiopharmaceuticals and treatment with somatostatin analogs (SSAs), including octreotide and octreotate. There are 5 subtypes of SSTRs, with subtypes 2, 3, and 5 most commonly expressed (4). ${ }^{111}$ In-diethylenetriamine pentaacetate-conjugated octreotide ( ${ }^{111}$ In-pentetreotide/ OctreoScan; Mallinckrodt Nuclear Medicine) was the first agent to receive U.S. Food and Drugs Administration approval (in 1994) for functional imaging of NENs with planar scintigraphy or SPECT (5). ${ }^{99 \mathrm{~m}}$ Tc-labeled SSAs, including the commercially available ${ }^{99 \mathrm{~m}}$ Tc-ethylenediaminediacetic acid hydrazinonicotinamide-[D$\mathrm{Phe}^{1}, \mathrm{Tyr}^{3}$-octreotide], were also developed to improve image quality with lower absorbed radiation dose (6). Newer ${ }^{68} \mathrm{Ga}$ - or ${ }^{64} \mathrm{Cu}$-tetraxetan (DOTA)-conjugated SSAs for PET have shown diagnostic performance superior to that of ${ }^{111}$ In-pentetreotide and are the current modality of choice for functional imaging $(5,7)$. Different DOTA peptides exist and have varying affinity for the SSTR subtypes (Table 1).

Management of NENs is based on the grade, subtype, distribution, and extent of disease. Anatomic imaging with CT and MRI is standard practice to assess disease location and extent, although 
TABLE 1

In Vitro Affinity of DOTA Peptides for Common SSTR Subtypes (5)

\begin{tabular}{cccc}
\hline Radiopeptide & SSTR-2 & SSTR-3 & SSTR-5 \\
\hline${ }^{111}$ In-DOTANOC & 2.9 & 8 & 11.2 \\
${ }^{111}$ In-DOTATATE & 1.5 & $>1,000$ & 547 \\
${ }^{111}$ In-DOTATOC & 4.6 & 120 & 130 \\
${ }^{68} \mathrm{Ga}-D O T A N O C$ & 1.9 & 40 & 7.2 \\
${ }^{68} \mathrm{Ga}$-DOTATATE & 0.2 & $>1,000$ & 377 \\
${ }^{68} \mathrm{Ga}$-DOTATOC & 2.5 & 613 & 73 \\
\hline
\end{tabular}

Data are half-maximal inhibitory concentration in nanomoles (lower values represent higher affinity).

radiopharmaceutical development has led to improvements in imaging and therapy (together termed theranostics). Initially, high-dose ${ }^{111}$ In-pentetreotide was used for therapy (8), via Auger electrons, although the efficacy was limited (9). The use of ${ }^{177} \mathrm{Lu}$ or ${ }^{90} \mathrm{Y}$ ( $\beta$-emitters) conjugated to SSAs with DOTA has been more effective (10). Specifically, ${ }^{177}$ Lu-DOTATATE-based peptide receptor radionuclide therapy (PRRT), studied in a phase 3, multicenter, randomized controlled trial (NETTER-1) in patients with inoperable or advanced and progressive midgut NENs, showed superior outcomes to standard-of-care therapy (10).

This paper reviews the current status and advances in imaging of NENs, with a focus on the use of SSTR PET with respect to PRRT.

\section{THE ROLE OF CONVENTIONAL IMAGING}

$\mathrm{CT}$ is commonly the initial imaging modality for evaluation of a suspected NEN. The detection rate of primary small-bowel NENs is about $50 \%(11,12)$. Metastatic mesenteric nodes are typically larger than the primary itself and are often calcified. When a small-bowel NEN is known or suspected, a negative oral contrast medium (methylcellulose, polyethylene glycol, or water) is preferred over a conventional radiopaque contrast medium, to avoid masking the primary enhancing lesion on the bowel wall $(13,14)$. Primary pancreatic NENs have a detection rate of about $80 \%-100 \%$ on CT (15). It is important to obtain an abdominal multiphase CT scan with intravenous contrast medium, since most pancreatic NENs and their hepatic metastases are arterially enhancing and occult on a single portal venous phase (Fig. 1) $(11,14,16)$. Around $22 \%$ of pancreatic NENs are arterially hypoenhancing, and in these cases the portal venous and delayed phases can help in detection $(11,17)$.

\section{NOTEWORTHY}

- SSTR PET can be used to reliably assess SSTR expression both visually and semiquantitatively.

- SSTR PET is essential for the proper assessment of eligibility for PRRT.

- SSTR expression is both a prognostic (correlates with outcome regardless of the therapy) and predictive (correlates specifically with response to PRRT) parameter for NENs.

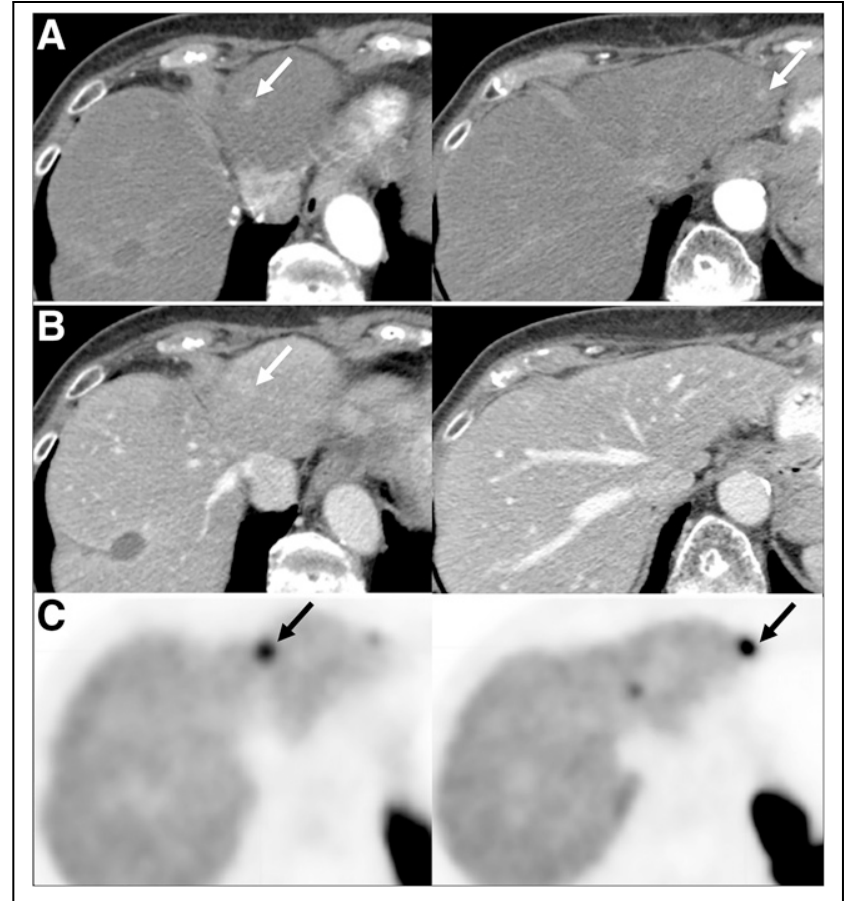

FIGURE 1. Pancreatic NEN with hepatic metastases (arrows). (A) Abdominal contrast-enhanced CT showing 2 small arterially enhancing left hepatic lesions. (B) Corresponding portal venous phase, where lesions are less conspicuous. (C) Transaxial PET images showing ${ }^{68} \mathrm{Ga}$-DOTATATE avidity in same lesions.

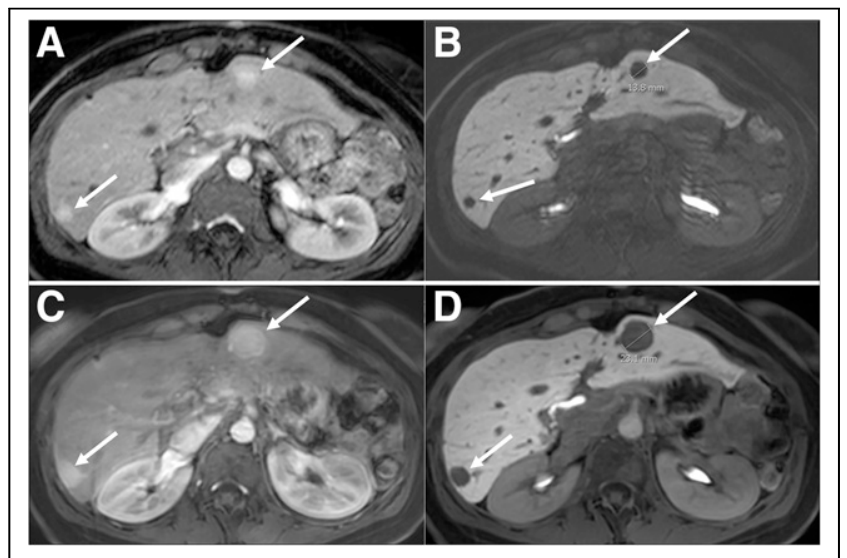

FIGURE 2. Abdominal contrast-enhanced MRI (with gadoxetate disodium) in patient with pancreatic NEN with hepatic metastases. (A and $B$ ) On arterial phase (A) and 20-min delayed-phase (B) images, 2 metastatic lesions (arrows) show arterial enhancement and contrast washout during delayed phase. (C and D) On arterial phase (C) and delayed-phase (D) images, size of previous lesions on 18-mo follow-up MRI has increased. Lesions are better delineated during delayed phase, facilitating accurate size measurements.

MRI is superior to CT for detecting hepatic metastases $(18,19)$. As with CT, multiphase MRI with intravenous contrast medium is recommended since most primary and metastatic NENs show arterial enhancement. Additionally, diffusion-weighted imaging and the delayed postcontrast phase using gadoxetic acid (hepato-specific paramagnetic contrast agent) are useful for detection of hepatic 
metastases. Hepatic metastases typically show a high signal on diffusion-weighted imaging (combination of T2 shine-through and true diffusion restriction), making them more conspicuous; this tool is especially helpful in patients with severe renal failure, for whom intravenous gadolinium is contraindicated $(19,20)$. The most sensitive tool for detection of hepatic metastases is the 20-min postcontrast delayed phase after intravenous administration of gadoxetic acid (Fig. 2), which is retained in hepatocytes but not in metastases, creating a high lesion-to-background contrast on the delayed image. In addition to having high sensitivity for lesion detection, the 20-min delayed phase allows for more accurate and reproducible measurement of baseline and follow-up lesion dimensions on imaging (20-23).

Findings on anatomic imaging associated with higher-grade tumors, which apply to both CT and MRI, include large tumor size ( $\geq 2 \mathrm{~cm}$ ), ill-defined margins, low or moderate arterial hyperenhancement, dilatation of the main pancreatic duct, vascular invasion, and presence of nodal or distant metastases; findings specific to MRI include nonintense T2 signal and, most importantly, high diffusion restriction (24,25). Several studies show that apparent diffusion coefficients inversely correlate with mitotic count and Ki-67 index. A significant difference in apparent diffusion coefficients has been observed between G1 and G2 tumors and between G1/G2 and G3 tumors, with suggested apparent diffusion coefficient cutoffs of below $0.95 \times 10^{-3}$ to $1.19 \times 10^{-3} \mathrm{~mm}^{2} / \mathrm{s}$ for G3 tumors $(18,19)$.

\section{PET RADIOPHARMACEUTICALS}

Introduced in $2001,{ }^{68} \mathrm{Ga}$-DOTATOC was the first PET-SSA ligand (26). As opposed to the SSTR-2-selective DOTATATE, DOTATOC retains an octreotide-like affinity profile (Table 1)

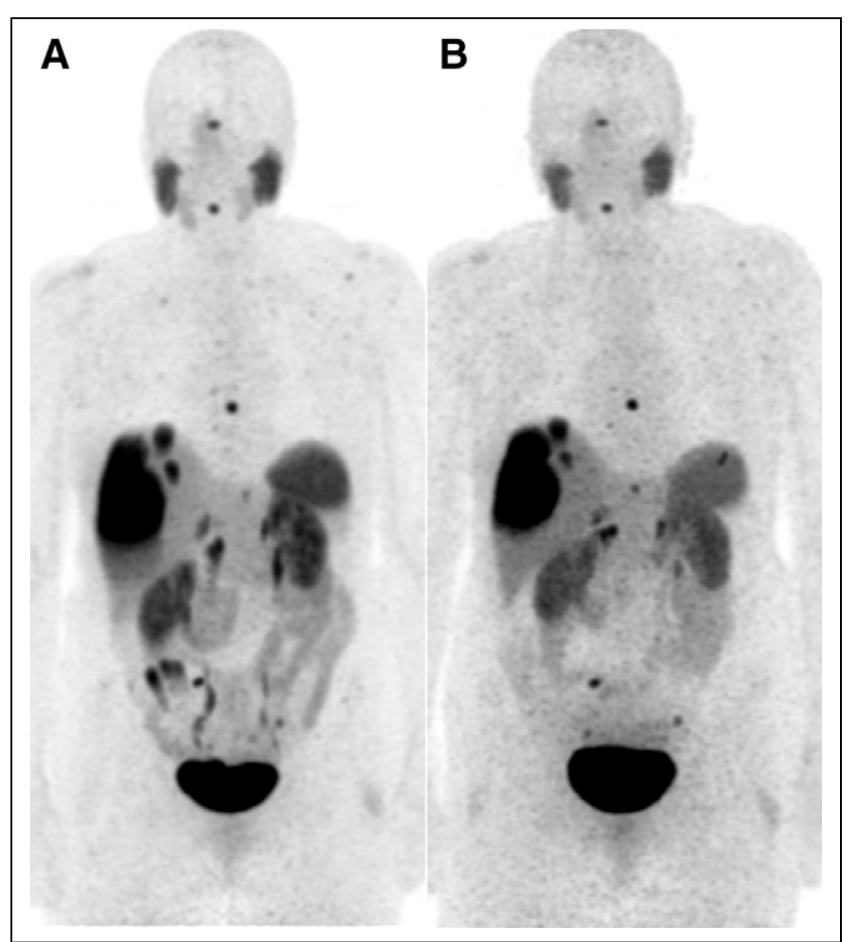

FIGURE 3. ${ }^{68} \mathrm{Ga-DOTATATE}(\mathrm{A})$ and ${ }^{64} \mathrm{Cu}$-DOTATATE (B) maximumintensity-projection PET images of metastatic NEN showing similar findings. Both studies were performed as part of PET/MRI, with uptake times for ${ }^{68} \mathrm{Ga}$-DOTATATE and ${ }^{64} \mathrm{Cu}$-DOTATATE being 113 and $118 \mathrm{~min}$, respectively (3 $\mathrm{min} /$ bed position for both).
(27). A comparison of ${ }^{68}$ Ga-DOTATOC to ${ }^{68}$ Ga-DOTATATE $\mathrm{PET} / \mathrm{CT}$ in the same patients showed a similar diagnostic accuracy, despite potential advantages for ${ }^{68} \mathrm{Ga}$-DOTATOC in the total number of detected lesions and a higher $\mathrm{SUV}_{\max }(28)$. Today, ${ }^{68} \mathrm{Ga}-$ DOTATOC and ${ }^{68} \mathrm{Ga}$-DOTATATE are the most commonly used radiopharmaceuticals for imaging NENs, with no clear superiority of either one of these compounds.

One of the main disadvantages of ${ }^{68} \mathrm{Ga}-\mathrm{SSA}$-based imaging is the high liver background and short radiopharmaceutical halflife. For the latter, newer SSTR radiopharmaceuticals, such as ${ }^{64} \mathrm{Cu}$-labeled SSA (Food and Drug Administration-approved in September 2020) may provide an advantage. Figure 3 shows the same patient imaged with the 2 different radioisotopes. Potential advantages of ${ }^{64} \mathrm{Cu}$ include its longer half-life (12.7 h vs. $68 \mathrm{~min}$ for ${ }^{68} \mathrm{Ga}$ ) and resultant higher target-to-background ratios on delayed imaging, as well as a shorter positron range in tissue (mean, $0.6 \mathrm{~mm}$, vs. $3.5 \mathrm{~mm}$ for ${ }^{68} \mathrm{Ga}$ ). These factors may result in better imaging characteristics, especially at later times (3-24 h after injection) (29). Conversely, ${ }^{64} \mathrm{Cu}$ has a significantly lower positron branching ratio $(0.17)$ than ${ }^{68} \mathrm{Ga}(0.89)$, which may degrade image quality or at least require a longer acquisition time. A prospective head-to-head comparison of ${ }^{64} \mathrm{Cu}$-DOTATATE and ${ }^{68} \mathrm{Ga}$-DOTATOC PET/CT in 59 subjects with NENs showed ${ }^{64} \mathrm{Cu}$-DOTATATE to be advantageous, detecting $83 \%$ of the true-positive lesions that were discordant between the radiopharmaceuticals (30). However, dual-time-point imaging with ${ }^{64} \mathrm{Cu}$-DOTATATE in 35 patients showed similar accuracy for 1-h and 3-h imaging (31), suggesting that the improved detection rate seen in the previous study was due to factors other than the target-to-background ratio. Notably, ${ }^{64} \mathrm{Cu}$-DOTA is prone to demetallation and transchelation in vivo, and better results may be expected with new sarcophagine-based chelators (32).

The SSAs discussed thus far are SSTR agonists, resulting in activation and internalization of the receptor on binding. Radiolabeled SSTR antagonists, such as ${ }^{68}$ Ga-DOTA-JR11, are characterized by a lack of internalization, rapid blood-pool clearance, and greater tumor uptake, aiding detection of metastases (33). A prospective head-to-head comparison between ${ }^{68}$ Ga-NODAGA-JR11, a SSTR antagonist, and ${ }^{68} \mathrm{Ga}$-DOTATOC PET/CT in 12 patients with NENs demonstrated that the favorable biodistribution of the antagonist resulted in a higher detection rate of hepatic metastases and a significantly greater lesion-based overall sensitivity ( $94 \%$ vs. $59 \%)(34)$.

When SSTR imaging is suboptimal, other PET agents have been developed to target different receptors overexpressed by the NENs, including the glucagon-like peptide 1 receptor ligand ${ }^{68}$ Ga-DOTA-exendin-4, which may facilitate the detection of benign insulinomas (frequently SSTR-negative) $(35,36)$. The CXCR-4 ligand ${ }^{68} \mathrm{Ga}$-pentixafor seems superior to conventional SSTR imaging for G3 NETs, but its role relative to ${ }^{18} \mathrm{~F}$-FDG PET remains to be determined (37). SSTR PET typically shows high uptake in well-differentiated or low-grade lesions and lower uptake in poorly differentiated or high-grade lesions. In the latter scenario, ${ }^{18}$ F-FDG PET is complementary in that it detects aggressive, poorly differentiated disease with higher grade and worse prognosis (Fig. 4; Supplemental Figs. 1 and 2; supplemental materials are available at http://jnm.snmjournals.org). No more than around $40 \%$ of patients with G1 disease are thought to have ${ }^{18}$ F-FDG uptake, whereas almost all patients with G3 disease have ${ }^{18}$ F-FDG uptake $(38-41)$. Since NENs are vastly heterogeneous and it would be impossible to sample all lesions in 


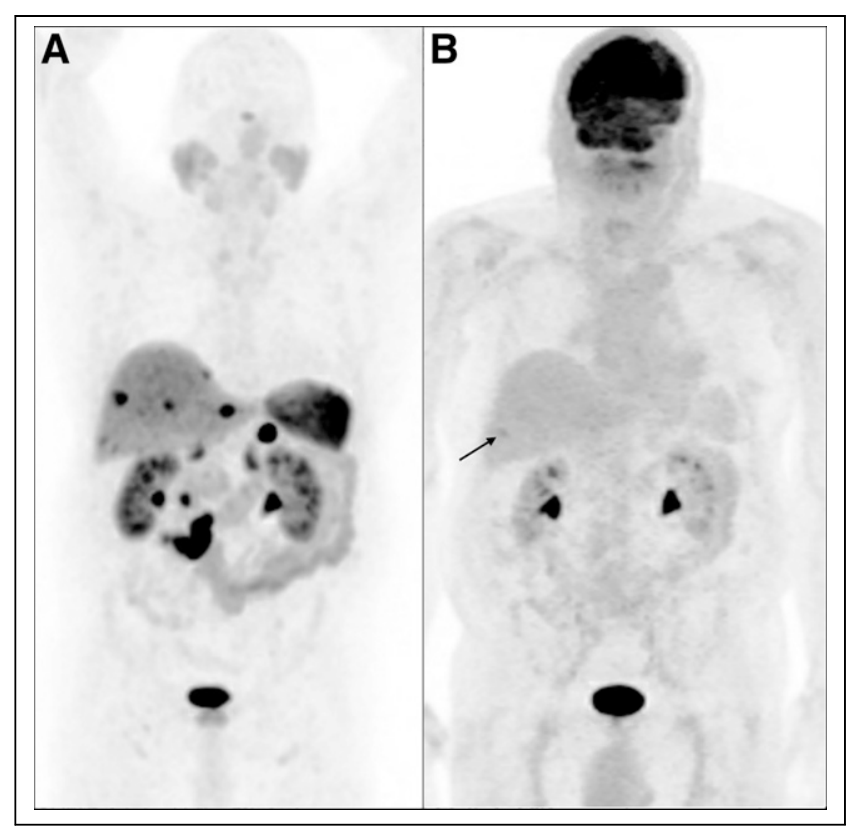

FIGURE 4. Maximum-intensity projection images of patient with metastatic grade 1 (Ki-67<2\%) NEN from small-bowel primary. (A) ${ }^{68} \mathrm{Ga}$-DOTATATE PET shows prominent uptake in primary tumor, lymphadenopathy, and liver metastases. (B) ${ }^{18}$ F-FDG PET shows no abnormal uptake (arrow points out incidentally noted fractured rib).

the body, the combination of SSTR and ${ }^{18}$ F-FDG PET provides a noninvasive understanding of disease heterogeneity and likelihood of PRRT response (42).

Currently, ${ }^{18}$ F-FDG PET is used for staging G3 disease and can be used to complement SSTR PET when Ki-67 is $10 \%$ or more $(41)$. Also, a positive ${ }^{18} \mathrm{~F}$-FDG PET result may be used to reconsider PRRT for a patient. Specifically, the combination of high SSTR and low ${ }^{18}$ F-FDG avidity increases the likelihood of benefit from PRRT; however, the ratio of differentiated to dedifferentiated disease at which PRRT ceases to be useful remains to be determined. In fact, it seems possible that in the event of marked uptake on SSTR PET with limited sites of ${ }^{18}$ F-FDG-avid disease, a combination of PRRT and targeted external radiation to the ${ }^{18} \mathrm{~F}-\mathrm{FDG}$-avid lesions may prolong survival. Ultimately, a combination of SSTR and ${ }^{18}$ F-FDG PET will likely provide a synergistic pictorial road map of disease for determining when to use PRRT, combination PRRT, and targeted external radiotherapy versus an alternative therapy (43-45).

\section{TUMOR QUANTIFICATION, CURRENT GUIDELINES, AND THE KRENNING SCALE}

SSTR PET can be used to assess SSTR expression visually and semiquantitatively. Cell membrane-based SSTR-2 expression on immunohistochemistry in NENs correlates with the SUVs on ${ }^{68}$ Ga-DOTATOC PET/CT (46). Some cases considered negative on immunohistochemistry demonstrated mild uptake on SSTR PET, possibly because of SSTR-5 binding or tumor heterogeneity. Campana et al. (47) suggested that the $\mathrm{SUV}_{\max }$ correlated with clinicopathologic features of NENs and could serve as a prognostic index, alongside anatomic location, primary tumor grade, and Ki-67 status. Velikyan et al. (48) reported that kinetic modeling parameters, rather than SUV, reflected receptor density more accurately based on absence of a linear correlation between SUV and net uptake rate in tumors with high SSTR expression. Specifically, SUVs correlated with receptor density at low values, with a nonlinear relationship thereafter leading to underestimation of receptor expression. Although this finding might reflect plasma peptide availability as a limiting factor for tracer uptake in patients with high SSTR expression and high tumor burden, an alternative explanation could be related to receptor saturation.

More recently, volumetric parameters have been evaluated in well-differentiated NENs (49). Specifically, the concept of SSTRexpressing tumor volume, representing the volume of tumor with more than $50 \% \mathrm{SUV}_{\max }$, and total-lesion SSTR expression, calculated as SSTR-expressing tumor volume $\times \mathrm{SUV}_{\text {mean }}$ in the volume of interest, have been defined. A sum of each of these volumetric parameters can be calculated; the literature suggests there may be a significant correlation between whole-body cumulative SSTRexpressing tumor volume and progression-free survival after PRRT. Nevertheless, estimation of tumor volume based on uptake will likely remain problematic given the intrinsic heterogeneity in tumoral SSTR expression.

Recent guidelines formulated under the auspices of the European Association of Nuclear Medicine recommend the use of ${ }^{68} \mathrm{Ga}-$ labeled SSAs in combination with CT or MRI for diagnosis, for staging, for restaging after surgery, for following progression, and for known or suspected NETs (50). The National Comprehensive Cancer Network guidelines recommend SSTR PET before PRRT for advanced NENs (51). Although a few studies using ${ }^{68}$ Ga-DOTATOC have suggested that $\mathrm{SUV}_{\max }$ thresholds be used to determine eligibility for PRRT - for example, $\mathrm{SUV}_{\max }$ cutoffs of 17.9 (52) and 16.5 (53) - differences between scanners and imaging techniques may produce slight variations, which make $\mathrm{SUV}_{\text {max }}$ problematic to use. An alternative is to use a tumor-to-liver ratio of 2.2 (53). The American College of Radiology practice parameters suggest visually assessed tumor uptake equal to or more than liver uptake as an eligibility criterion for PRRT (54).

The Krenning score was developed using ${ }^{111}$ In-pentetreotide scintigraphy (8) and has been extrapolated to SSTR PET (modified Krenning score). A 5-point scale has been proposed on the basis of a qualitative assessment of lesion uptake relative to blood pool and hepatic activity, where 0 is no uptake, 1 is very low uptake, 2 is uptake no more than in the liver, 3 is uptake greater than in the liver, and 4 is uptake greater than in the spleen (55). However, the relationship between the Krenning score from ${ }^{111}$ In-pentetreotide scintigraphy and the modified Krenning score from SSTR PET is limited (56). Disease has a bias toward higher scores on SSTR PET than on ${ }^{111}$ In-pentetreotide scintigraphy (Supplemental Fig. 3). Part of this bias is due to differences in equipment (higher sensitivity of PET vs. planar scintigraphy or SPECT) and imaging time points ( ${ }^{111}$ In-pentetreotide scintigraphy at $24 \mathrm{~h}$ after injection vs. SSTR PET at $1 \mathrm{~h}$ after injection).

Although there are few formal data to support the use of SSTR PET over ${ }^{111}$ In-pentetreotide scintigraphy, SSTR PET has become the standard for pre-PRRT patient selection because of its higher sensitivity, faster imaging times, and lower radiation dose. For lesions larger than $2 \mathrm{~cm}$, it is appropriate to use the modified Krenning score, and PRRT should be considered with a score of 3 or 4 . Caution should be used before treating patients with lesions smaller than $2 \mathrm{~cm}$ with a modified Krenning score of 3 or 4, as these patients are unlikely to have fulfilled criteria if imaged with ${ }^{111}$ In-pentetreotide. This is to emphasize that the current data do not provide 
sufficient evidence for the use of SSTR PET in this setting. PRRT should not be considered when lesions show no or low uptake on SSTR PET.

\section{REPORTING SSTR PET}

There is a need for standardized interpretation of SSTR PET given that findings on baseline imaging partly determine treatment success with radioligand therapies (57). The report (Supplemental Fig. 4) should include a concise clinical history, including NEN subtype, tumor grade and differentiation, and prior treatments (medical or surgical). The imaging parameters, in terms of the specific radiopeptide and its administered activity, uptake time, duration of imaging (time per bed position), and area imaged, should be documented. Comparison and correlation with any prior SSTR imaging, ${ }^{18} \mathrm{~F}$ FDG PET, and other anatomic imaging should be performed. Findings should detail the site and size of the lesions (the latter if seen on corresponding CT/MRI) and uptake intensity, which can be expressed semiquantitatively (commonly as $\mathrm{SUV}_{\max }$ ). The pattern of tracer uptake (intralesional heterogeneity) and assessment of lesion resectability (i.e., relation with vascular and major structures) may be helpful to further guide management. The conclusion should provide the modified Krenning score, and additional diagnostic examinations or follow-up can be suggested.

The NETPET score is a grading system that combines findings on SSTR and ${ }^{18}$ F-FDG PET with a single parameter (58). This scoring system has been developed as a prognostic biomarker. Although rarely included in reports since SSTR and ${ }^{18}$ F-FDG PET are not routinely performed together, its rate of inclusion may change in the future.

The SSTR reporting and data systems (RADS) has also been introduced as part of the umbrella molecular imaging RADS, a 5-point scale (from 1 [no evidence of disease and definitely benign] to 5 [high certainty of NEN]) indicating both disease site and radiotracer avidity (55). SSTR RADS entails a 3-point qualitative scoring of uptake level, where up to 5 target (largest, most avid) lesions can be identified, with overall score defined as the highest scored lesion. A summed RADS score, including all 5 target lesions, has also been suggested (59). Future validation of this framework is warranted, including inter- and intraobserver agreement studies and histopathology correlation.

\section{Disease Burden, Outcome Prediction, and Response Assessment}

SSTR expression is both a prognostic (correlates with outcome regardless of therapy) and predictive (correlates specifically with response to PRRT) parameter for NENs. (60). The current literature suggests that higher baseline SUVs on SSTR PET predict better post-PRRT outcomes. Öksüz et al. (52) reported that high pretherapy primary tumor uptake suggested a good response to PRRT; Kratochwil et al. (53) reported that high pretherapy uptake in liver metastases suggested a good response; and Ambrosini et al. (60) reported better outcomes in patients with high baseline SUVs. To avoid scannerrelated variations, parameters such as tumor-to-liver and tumor-to spleen ratios may be used. It has been reported that a tumor-to-liver ratio of more than 2.2 is predictive of a favorable response. It has, however, been demonstrated that a high uptake (e.g., Krenning grade 4) is associated with response to PRRT in only $60 \%$ of patients $(61)$.

The literature on response evaluation is more variable, and we are only beginning to understand how post-therapy SSTR PET correlates with endpoints such as time to progression, progression-free, and overall survival. Haug et al. (62) studied SUV $\max$ and tumorto-spleen ratio for prediction of time to progression and clinical outcome after a first PRRT cycle in well-differentiated NENs. The authors found that reduced uptake after therapy predicted time to progression and correlated with clinical improvement. Further, interval change in tumor-to-spleen ratio was superior to interval change in $\mathrm{SUV}_{\max }$. Meanwhile, Gabriel et al. (6) reported essentially random SUV fluctuations after PRRT. The question remains: Does diminishing tumoral radiotracer uptake reflect true disease improvement or is there a higher degree of tumor dedifferentiation with loss of SSTR expression? Accordingly, the recently updated appropriateuse criteria (63) for SSTR PET notes that response should be assessed by the disappearance of known lesions or development of new lesions, rather than changes in SUVs.

Monitoring response to PRRT with SSTR PET and attempting to interpret the biologic significance of tumor uptake change are challenging. One study evaluated 46 patients with advanced NENs treated with 2-7 cycles of PRRT and compared the results from the post-therapy ${ }^{68}$ Ga-DOTATATE PET to CT/MRI with RECIST. The authors found little advantage to SSTR PET over conventional imaging for response assessment (6). In another study, of 66 patients, ${ }^{68} \mathrm{Ga}$-DOTATOC and ${ }^{18} \mathrm{~F}$-FDG PET was done at baseline, at $3 \mathrm{mo}$, and again at 6-9 mo after completion of PRRT. The authors concluded that uptake on ${ }^{18}$ F-FDG PET at baseline and follow-up had a stronger correlation with the outcome than did SSTR PET and that combination imaging with both radiopharmaceuticals might be advisable across all tumor grades (43).

Also, a high overall tumor burden and tumor heterogeneity on SSTR PET is likely to be associated with worse prognosis. SSTR PET helps in assessing the heterogeneity of NENs that exist at the interpatient, intrapatient, interlesional level at a specific time point or longitudinally at different time points. This heterogeneity implies a variety of cells displaying variable characteristics in terms of metabolism, proliferation, metastatic potential, and therapy response. Distinct metastases may harbor different cellular clones with varying SSTR expression. The primary tumor and its metastases may also differ. Indeed, this may impact the chance of PRRT success and explains why cure is rarely possible with systemic metastatic disease. In a study by Graf et al. (64), only patients with at least $90 \%$ of metastases positive for SSTR were treated with PRRT. Positive lesions were viewed in 3 dimensions,

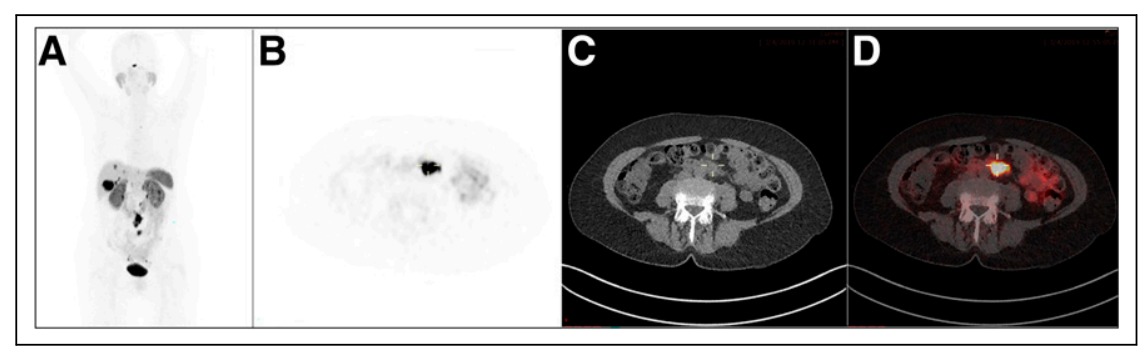

FIGURE 5. A 60-y-old woman with small-bowel NET on octreotide therapy. ${ }^{68}$ Ga-DOTATATE PET/ CT (maximum-intensity projection [A], transaxial PET [B], CT [C], PET/CT [D]) shows prominent uptake at tumor sites. This finding would make patient eligible for PRRT, but overall limited extent of disease in liver and retroperitoneum favors surgical resection over PRRT. 
and a lesion that had a change in score from 3 or 4 to 2 , or from 2 to 1 , that persisted over more than $5 \mathrm{~mm}$ in any plane was defined as heterogeneous. Only the solid portion of a necrotic lesion was assessed. If more than $50 \%$ of lesions were deemed heterogeneous, the patient was labeled as heterogeneous. This study confirmed that heterogeneity had a negative impact on overall survival and time to progression after PRRT. Indeed, heterogeneity surpassed $\mathrm{Ki}-67$ as a prognostic marker, especially related to PRRT, reinforcing the suspicion that PRRT may target the less aggressive, SSTR-positive cells, sparing the rest. Thus, even when decreased tumor size suggests response by RECIST, the more aggressive cells might remain viable. These observations highlight an intrinsic flaw of using quantitative parameters such as $\mathrm{SUV}_{\max }$ alone, which do not account for the intralesional variation in SSTR expression. Interestingly, some authors have observed that, after PRRT, heterogeneous lesions may become more homogeneous. In the future, use of textural characteristics such as entropy and skewness may prove superior to our current methodology for lesion analysis.

Recently, a prospective study on 158 patients divided into 3 independent ${ }^{177}$ Lu-PRRT cohorts demonstrated that specific circulating tumor transcripts (messenger RNA) specifically predict the outcome of PRRT and therefore represent a marker of radiosensitivity (65), whereas the circulating transcript signature NETest allows accurate monitoring of the course of disease during treatment and integrates with imaging (66).

The primary site of the tumor, which can often be elucidated with SSTR PET, is a prognostic factor and should be incorporated in the decision algorithm for PRRT. Midgut and pancreatic NENs are included in the Food and Drug Administration-approved indications for PRRT. Bronchial NENs represent a special category, with typical tumors considered more appropriate for PRRT because of higher SSTR expression. In the case of a pheochromocytoma or paraganglioma, the current recommendation reserves PRRT for metaiodobenzylguanidine-negative tumors only, for which ${ }^{131}$ I-metaiodobenzylguanidine treatment is precluded. The distribution and extent of disease, ideally evaluated with SSTR PET, also affects management. In general, caution is needed in tumors with extensive mesenteric and peritoneal involvement, since PRRT may increase the risk of complications from a desmoplastic reaction. As the tumors metastasize, the total tumor burden may play a role, depending on the primary site of disease. For example, pancreatic NENs with more than $25 \%$ liver involvement and bone metastases have worse prognosis, whereas gastric NENs show no significant difference in outcome based on distribution (67). In general, tumor burden is termed limited if fewer than 5 lesions are detected at 1 site, moderate if more than 5 lesions at 2 sites, and extensive if more than 2 sites are involved, and this affects the treatment approach (Fig. 5). Most gastroenteropancreatic NENs present with hepatic metastases at diagnosis despite low Ki-67, and the presence of hepatic metastases profoundly decreases overall survival. PRRT may be helpful for nonresectable hepatic metastases and indeed may render the lesions resectable. In liver-dominant disease, intraarterial PRRT is being investigated.

\section{CONCLUSION}

SSTR PET is the preferred imaging modality at initial diagnosis of low- and intermediate-grade NENs, especially for localization of the primary tumor and determining disease extent. SSTR PET is essential for selecting patients for PRRT, whereas its role in response monitoring is still being evaluated. Although SSTR expression can be assessed visually and semiquantitatively, with various suggested thresholds, a modified Krenning score is used in current clinical practice.

\section{DISCLOSURE}

No potential conflict of interest relevant to this article was reported.

\section{REFERENCES}

1. Cives M, Strosberg JR. Gastroenteropancreatic neuroendocrine tumors. CA Cancer J Clin. 2018;68:471-487.

2. Rindi G, Klimstra DS, Abedi-Ardekani B, et al. A common classification framework for neuroendocrine neoplasms: an International Agency for Research on Cancer (IARC) and World Health Organization (WHO) expert consensus proposal. Mod Pathol. 2018;31:1770-1786.

3. Nagtegaal ID, Odze RD, Klimstra D, et al. The 2019 WHO classification of tumours of the digestive system. Histopathology. 2020;76:182-188.

4. Krenning EP, Kwekkeboom DJ, Bakker WH, et al. Somatostatin receptor scintigra-

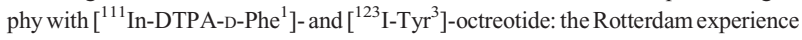
with more than 1000 patients. Eur J Nucl Med. 1993;20:716-731.

5. Rufini V, Calcagni ML, Baum RP. Imaging of neuroendocrine tumors. Semin Nucl Med. 2006;36:228-247.

6. Gabriel M, Oberauer A, Dobrozemsky G, et al. ${ }^{68}$ Ga-DOTA-Tyr ${ }^{3}$-octreotide PET for assessing response to somatostatin-receptor-mediated radionuclide therapy. $\mathrm{J} \mathrm{Nucl}$ Med. 2009;50:1427-1434

7. Buchmann I, Henze M, Engelbrecht S, et al. Comparison of ${ }^{68} \mathrm{Ga}$-DOTATOC PET and ${ }^{111}$ In-DTPAOC (Octreoscan) SPECT in patients with neuroendocrine tumours. Eur J Nucl Med Mol Imaging. 2007;34:1617-1626.

8. Krenning EP, Valkema R, Kooij PP, et al. Scintigraphy and radionuclide therapy with [indium-111-labelled-diethyl triamine penta-acetic acid-D-Phe1]-octreotide. Ital J Gastroenterol Hepatol. 1999;31(suppl 2):S219-S223.

9. Kwekkeboom D, Krenning EP, de Jong M. Peptide receptor imaging and therapy. $J$ Nucl Med. 2000;41:1704-1713.

10. Kunikowska J, Królicki L, Hubalewska-Dydejczyk A, Mikołajczak R, Sowa-Staszczak A, Pawlak D. Clinical results of radionuclide therapy of neuroendocrine tumours with ${ }^{90} \mathrm{Y}$-DOTATATE and tandem ${ }^{90} \mathrm{Y} /{ }^{177} \mathrm{Lu}$-DOTATATE: which is a better therapy option? Eur J Nucl Med Mol Imaging. 2011;38:1788-1797.

11. Maxwell JE, O'Dorisio TM, Howe JR. Biochemical diagnosis and preoperative imaging of gastroenteropancreatic neuroendocrine tumors. Surg Oncol Clin N Am. 2016;25:171-194.

12. Dahdaleh FS, Lorenzen A, Rajput M, et al. The value of preoperative imaging in small bowel neuroendocrine tumors. Ann Surg Oncol. 2013;20:1912-1917.

13. Woodbridge LR, Murtagh BM, Yu DF, Planche KL. Midgut neuroendocrine tumors: imaging assessment for surgical resection. Radiographics. 2014;34:413-426.

14. Sahani DV, Bonaffini PA, Fernández-Del Castillo C, Blake MA. Gastroenteropancreatic neuroendocrine tumors: role of imaging in diagnosis and management. Radiology. 2013;266:38-61.

15. Kuo JH, Lee JA, Chabot JA. Nonfunctional pancreatic neuroendocrine tumors. Surg Clin North Am. 2014;94:689-708.

16. Bushnell DL, Baum RP. Standard imaging techniques for neuroendocrine tumors. Endocrinol Metab Clin North Am. 2011;40:153-162.

17. Worhunsky DJ, Krampitz GW, Poullos PD, et al. Pancreatic neuroendocrine tumours: hypoenhancement on arterial phase computed tomography predicts biological aggressiveness. $H P B$ (Oxford). 2014;16:304-311.

18. Dromain C, de Baere T, Lumbroso J, et al. Detection of liver metastases from endocrine tumors: a prospective comparison of somatostatin receptor scintigraphy, computed tomography, and magnetic resonance imaging. J Clin Oncol. 2005;23:70-78.

19. Yu R, Wachsman A. Imaging of neuroendocrine tumors: indications, interpretations, limits, and pitfalls. Endocrinol Metab Clin North Am. 2017;46:795-814.

20. Shimada K, Isoda H, Hirokawa Y, Arizono S, Shibata T, Togashi K. Comparison of gadolinium-EOB-DTPA-enhanced and diffusion-weighted liver MRI for detection of small hepatic metastases. Eur Radiol. 2010;20:2690-2698.

21. Ba-Ssalamah A, Uffmann M, Saini S, Bastati N, Herold C, Schima W. Clinical value of MRI liver-specific contrast agents: a tailored examination for a confident noninvasive diagnosis of focal liver lesions. Eur Radiol. 2009;19:342-357.

22. Qian HF, Zhu YM, Wu X, Li FQ, Xuan HB, Shen J. Comparison of enhanced magnetic resonance and diffusion-weighted imaging for detection of hepatic metastases [in Chinese]. Chung Kuo I Hsueh Ko Hsueh Yuan Hsueh Pao. 2012;34:621-624.

23. Giesel FL, Kratochwil C, Mehndiratta A, et al. Comparison of neuroendocrine tumor detection and characterization using DOTATOC-PET in correlation with contrast enhanced CT and delayed contrast enhanced MRI. Eur JRadiol. 2012;81:2820-2825. 
24. Besa C, Ward S, Cui Y, Jajamovich G, Kim M, Taouli B. Neuroendocrine liver metastases: value of apparent diffusion coefficient and enhancement ratios for characterization of histopathologic grade. J Magn Reson Imaging. 2016;44:1432-1441.

25. Kim M, Kang TW, Kim YK, et al. Pancreatic neuroendocrine tumour: correlation of apparent diffusion coefficient or WHO classification with recurrence-free survival. Eur J Radiol. 2016;85:680-687.

26. Hofmann M, Maecke H, Börner R, et al. Biokinetics and imaging with the somatostatin receptor PET radioligand ${ }^{68}$ Ga-DOTATOC: preliminary data. Eur J Nucl Med. 2001;28:1751-1757.

27. Reubi JCSJ, Waser B, Wenger S, Heppeler A, Schmitt JS, Mäcke HR. Affinity profiles for human somatostatin receptor subtypes SST1-SST5 of somatostatin radiotracers selected for scintigraphic and radiotherapeutic use. Eur J Nucl Med. 2000; 27:273-282.

28. Poeppel TD, Binse I, Petersenn S, et al. ${ }^{68} \mathrm{Ga}$-DOTATOC versus ${ }^{68} \mathrm{Ga}$-DOTATATE PET/CT in functional imaging of neuroendocrine tumors. J Nucl Med. 2011;52: 1864-1870.

29. Pfeifer A, Knigge U, Binderup T, et al. ${ }^{64} \mathrm{Cu}$-DOTATATE PET for neuroendocrine tumors: a prospective head-to-head comparison with ${ }^{111}$ In-DTPA-octreotide in 112 patients. J Nucl Med. 2015;56:847-854.

30. Johnbeck CB, Knigge U, Loft A, et al. Head-to-head comparison of ${ }^{64} \mathrm{Cu}$-DOTATATE and ${ }^{68} \mathrm{Ga}$-DOTATOC PET/CT: a prospective study of 59 patients with neuroendocrine tumors. J Nucl Med. 2017;58:451-457.

31. Loft M, Carlsen EA, Johnbeck CB, et al. Cu-DOTATATE PET in patients with neuroendocrine neoplasms: prospective, head-to-head comparison of imaging at 1 hour and 3 hours after injection. $J$ Nucl Med. 2021;62:73-80.

32. Hicks RJ, Jackson P, Kong G, et al. Cu-SARTATE PET imaging of patients with neuroendocrine tumors demonstrates high tumor uptake and retention, potentially allowing prospective dosimetry for peptide receptor radionuclide therapy. $\mathrm{J} \mathrm{Nucl} \mathrm{Med}$. 2019;60:777-785.

33. Krebs S, Pandit-Taskar N, Reidy D, et al. Biodistribution and radiation dose estimates for ${ }^{68}$ Ga-DOTA-JR11 in patients with metastatic neuroendocrine tumors. Eur J Nucl Med Mol Imaging. 2019;46:677-685.

34. Nicolas GP, Schreiter N, Kaul F, et al. Sensitivity comparison of ${ }^{68} \mathrm{Ga}-\mathrm{OPS} 202$ and ${ }^{68} \mathrm{Ga}$-DOTATOC PET/CT in patients with gastroenteropancreatic neuroendocrine tumors: a prospective phase II imaging study. J Nucl Med. 2018;59:915-921.

35. Antwi K, Fani M, Heye T, et al. Comparison of glucagon-like peptide-1 receptor (GLP-1R) PET/CT, SPECT/CT and 3T MRI for the localisation of occult insulinomas: evaluation of diagnostic accuracy in a prospective crossover imaging study. Eur J Nucl Med Mol Imaging. 2018;45:2318-2327.

36. Parihar AS, Vadi SK, Kumar R, et al. ${ }^{68} \mathrm{Ga}$ DOTA-exendin PET/CT for detection of insulinoma in a patient with persistent hyperinsulinemic hypoglycemia. Clin Nucl Med. 2018;43:e285-e286.

37. Werner RA, Weich A, Higuchi T, et al. Imaging of chemokine receptor 4 expression in neuroendocrine tumors: a triple tracer comparative approach. Theranostics. 2017; 7:1489-1498.

38. Garin E, Le Jeune F, Devillers A, et al. Predictive value of ${ }^{18}$ F-FDG PET and somatostatin receptor scintigraphy in patients with metastatic endocrine tumors. $J$ Nucl Med. 2009;50:858-864.

39. Binderup T, Knigge U, Loft A, Federspiel B, Kjaer A. ${ }^{18}$ F-fluorodeoxyglucose positron emission tomography predicts survival of patients with neuroendocrine tumors. Clin Cancer Res. 2010;16:978-985.

40. Johnbeck CB, Knigge U, Langer SW, et al. Prognostic value of ${ }^{18}$ F-FLT PET in patients with neuroendocrine neoplasms: a prospective head-to-head comparison with ${ }^{18}$ F-FDG PET and Ki-67 in 100 patients. $J$ Nucl Med. 2016;57:1851-1857.

41. Ezziddin S, Adler L, Sabet A, et al. Prognostic stratification of metastatic gastroenteropancreatic neuroendocrine neoplasms by ${ }^{18} \mathrm{~F}$-FDG PET: feasibility of a metabolic grading system. J Nucl Med. 2014;55:1260-1266.

42. Bahri H, Laurence L, Edeline J, et al. High prognostic value of ${ }^{18} \mathrm{~F}$-FDG PET for metastatic gastroenteropancreatic neuroendocrine tumors: a long-term evaluation. $\mathrm{J} \mathrm{Nucl}$ Med. 2014;55:1786-1790.

43. Nilica B, Waitz D, Stevanovic V, et al. Direct comparison of ${ }^{68}$ Ga-DOTA-TOC and ${ }^{18} \mathrm{~F}$-FDG PET/CT in the follow-up of patients with neuroendocrine tumour treated with the first full peptide receptor radionuclide therapy cycle. Eur J Nucl Med Mol Imaging. 2016;43:1585-1592.

44. Sansovini M, Severi S, Ianniello A, et al. Long-term follow-up and role of FDG PET in advanced pancreatic neuroendocrine patients treated with ${ }^{177} \mathrm{Lu}-\mathrm{D}$ OTATATE. Eur J Nucl Med Mol Imaging. 2017;44:490-499.
45. Hofman MS, Michael M, Kashyap R, Hicks RJ. Modifying the poor prognosis associated with ${ }^{18} \mathrm{~F}$-FDG-avid NET with peptide receptor chemo-radionuclide therapy (PRCRT). J Nucl Med. 2015;56:968-969.

46. Miederer M, Seidl S, Buck A, et al. Correlation of immunohistopathological expression of somatostatin receptor 2 with standardised uptake values in ${ }^{68} \mathrm{Ga}$-DOTATOC PET/CT. Eur J Nucl Med Mol Imaging. 2009;36:48-52.

47. Campana D, Ambrosini V, Pezzilli R, et al. Standardized uptake values of ${ }^{68} \mathrm{Ga}-$ DOTANOC PET: a promising prognostic tool in neuroendocrine tumors. $\mathrm{J} \mathrm{Nucl}$ Med. 2010;51:353-359.

48. Velikyan I, Sundin A, Sörensen J, et al. Quantitative and qualitative intrapatient comparison of ${ }^{68} \mathrm{Ga}$-DOTATOC and ${ }^{68} \mathrm{Ga}$-DOTATATE: net uptake rate for accurate quantification. J Nucl Med. 2014;55:204-210.

49. Toriihara A, Baratto L, Nobashi T, et al. Prognostic value of somatostatin receptor expressing tumor volume calculated from ${ }^{68} \mathrm{Ga}$-DOTATATE PET/CT in patients with well-differentiated neuroendocrine tumors. Eur J Nucl Med Mol Imaging. 2019;46:2244-2251.

50. Ambrosini V, Kunikowska J, Baudin E, et al. Consensus on molecular imaging and theranostics in neuroendocrine neoplasms. Eur J Cancer. 2021;146:56-73.

51. Shah MH, Goldner WS, Halfdanarson TR, et al. NCCN guidelines insights: neuroendocrine and adrenal tumors, version 2.2018. J Natl Compr Canc Netw. 2018;16:693702 .

52. Öksüz MÖ, Winter L, Pfannenberg C, et al. Peptide receptor radionuclide therapy of neuroendocrine tumors with ${ }^{90} \mathrm{Y}$-DOTATOC: is treatment response predictable by pre-therapeutic uptake of ${ }^{68} \mathrm{Ga}$-DOTATOC? Diagn Interv Imaging. 2014;95:289300 .

53. Kratochwil C, Stefanova M, Mavriopoulou E, et al. SUV of $\left[{ }^{68} \mathrm{Ga}\right]$ DOTATOC-PET/ $\mathrm{CT}$ predicts response probability of PRRT in neuroendocrine tumors. Mol Imaging Biol. 2015; 17:313-318.

54. Subramaniam RM, Bradshaw ML, Lewis K, Pinho D, Shah C, Walker RC. ACR practice parameter for the performance of gallium-68 DOTATATE PET/CT for neuroendocrine tumors. Clin Nucl Med. 2018;43:899-908.

55. Werner RA, Solnes LB, Javadi MS, et al. SSTR-RADS version 1.0 as a reporting system for SSTR PET imaging and selection of potential PRRT candidates: a proposed standardization framework. J Nucl Med. 2018;59:1085-1091.

56. Hope TA, Calais J, Zhang L, Dieckmann W, Millo C. ${ }^{111}$ In-pentetreotide scintigraphy versus 68 Ga-DOTATATE PET: impact on Krenning scores and effect of tumor burden. J Nucl Med. 2019;60:1266-1269.

57. Werner RA, Bundschuh RA, Bundschuh L, et al. Novel structured reporting systems for theranostic radiotracers. J Nucl Med. 2019;60:577-584.

58. Chan DL, Pavlakis N, Schembri GP, et al. Dual somatostatin receptor/FDG PET/CT imaging in metastatic neuroendocrine tumours: proposal for a novel grading scheme with prognostic significance. Theranostics. 2017;7:1149-1158.

59. Werner RA, Thackeray JT, Pomper MG, et al. Recent updates on Molecular Imaging Reporting and Data Systems (MI-RADS) for theranostic radiotracers-navigating pitfalls of SSTR- and PSMA-targeted PET/CT. J Clin Med. 2019;8:1060.

60. Ambrosini V, Campana D, Polverari G, et al. Prognostic value of ${ }^{68} \mathrm{Ga}$-DOTANOC PET/CT SUVmax in patients with neuroendocrine tumors of the pancreas. $\mathrm{J} \mathrm{Nucl}$ Med. 2015;56:1843-1848.

61. Kwekkeboom DJ, Kam BL, van Essen M, et al. Somatostatin-receptor-based imaging and therapy of gastroenteropancreatic neuroendocrine tumors. Endocr Relat Cancer. 2010;17:R53-R73.

62. Haug AR, Auernhammer CJ, Wängler B, et al. ${ }^{68}$ Ga-DOTATATE PET/CT for the early prediction of response to somatostatin receptor-mediated radionuclide therapy in patients with well-differentiated neuroendocrine tumors. J Nucl Med. 2010;51: 1349-1356.

63. Hope TA, Bergsland EK, Bozkurt MF, et al. Appropriate use criteria for somatostatin receptor PET imaging in neuroendocrine tumors. J Nucl Med. 2018;59:66-74.

64. Graf J, Pape UF, Jann H, et al. Prognostic significance of somatostatin receptor heterogeneity in progressive neuroendocrine tumor treated with Lu-177 DOTATOC or Lu-177 DOTATATE. Eur J Nucl Med Mol Imaging. 2020;47:881-894.

65. Bodei L, Kidd MS, Singh A, et al. PRRT genomic signature in blood for prediction of ${ }^{177}$ Lu-octreotate efficacy. Eur J Nucl Med Mol Imaging. 2018;45:1155-1169.

66. Bodei L, Kidd MS, Singh A, et al. PRRT neuroendocrine tumor response monitored using circulating transcript analysis: the NETest. Eur J Nucl Med Mol Imaging. 2020; 47:895-906.

67. Pu N, Habib JR, Bejjani M, et al. The effect of primary site, functional status and treatment modality on survival in gastroenteropancreatic neuroendocrine neoplasms with synchronous liver metastasis: a US population-based study. Ann Transl Med. 2021;9:329. 